\title{
When Things Are Better or Worse than Expected: The Medial Frontal Cortex and the Allocation of Processing Resources
}

\author{
Geoffrey F. Potts ${ }^{1}$, Laura E. Martin ${ }^{1}$, Philip Burton ${ }^{1}$, \\ and $P$. Read Montague ${ }^{2}$
}

\begin{abstract}
Access to limited-capacity neural systems of cognitive control must be restricted to the most relevant information. How the brain identifies and selects items for preferential processing is not fully understood. Anatomical models often place the selection mechanism in the medial frontal cortex (MFC), and one computational model proposes that the mesotelencephalic dopamine (DA) system, via its reward prediction properties, provides a "gate" through which information gains access to limited-capacity systems. There is a medial frontal eventrelated potential (ERP) index of attention selection, the anterior positivity (P2a), associated with DA reward system input to the MFC for the identification of task-relevant perceptual representations. The P2a has a similar spatio-temporal distribution as
\end{abstract}

\section{INTRODUCTION}

The brain contains limited-capacity information processing systems, particularly systems involved with cognitive control, which can only process a subset of available information at any given time. Effective generation of goaldirected behavior requires that access to limited-capacity systems be restricted to the most motivationally relevant information currently available. Attention is the cognitive operation by which representations are selected for preferential processing. Attention may provide access to working memory, and attended items and the contents of working memory may comprise the content of conscious awareness (reviewed in Parasuraman, 1998). However, the nature of the neural systems that identify and select items for preferential processing is an unresolved question.

One influential model of attention proposed by Posner and Dehaene (1994) and Posner and Petersen (1990) posits separate neural systems supporting distinct types of attention, including an anterior system located in the anterior cingulate cortex for the detection of task-relevant items, items that are targets in the context of the current

${ }^{1}$ Rice University, Houston, TX, ${ }^{2}$ Baylor College of Medicine, Houston, TX the medial frontal negativity (MFN), elicited to error responses or choices resulting in monetary loss. The MFN has also been linked to DA projections to the MFC but for action monitoring rather than attention selection. This study proposes that the P2a and the MFN reflect the same MFC evaluation function and use a passive reward prediction design containing neither instructed attention nor response to demonstrate that the ERP over medial frontal leads at the $\mathrm{P} 2 \mathrm{a} / \mathrm{MFN}$ latency is consistent with activity of midbrain DA neurons, positive to unpredicted rewards and negative when a predicted reward is withheld. This result suggests that MFC activity is regulated by DA reward system input and may function to identify items or actions that exceed or fail to meet motivational prediction.

task. However, this model does not describe how the anterior system distinguishes between relevant and irrelevant representations (i.e., how individual perceptual representations are identified as being relevant in the context of the individual's current goals). Some models of attention and working memory include a "supervisory" mechanism to control access to cognitive subsystems (e.g., Norman \& Shallice, 1986), but the exact computational operations or neural location of the supervisor is not defined.

One recent model suggests that controlled processing is engaged when well-rehearsed, automatic stimulusresponse mappings are insufficient to meet current task demands (Miller \& Cohen, 2001). The frontal cortex performs this control by maintaining goal representations and action plans for meeting those goals and providing a biasing signal that strengthens the appropriate perceptual representations and motor programs. The model suggests that the mesotelencephalic dopamine (DA) system, with its source in the ventral tegmental area (VTA) and widely distributed projections to the limbic system and the neocortex, particularly the medial frontal cortex (MFC: note that here we use MFC to refer to both the anterior cingulate cortex and the immediately surrounding neocortex), may provide a "gate" via which motivationally 
relevant information gains access to limited-capacity control systems in the prefrontal cortex (PFC) (Cohen, Braver, \& Brown, 2002; Braver \& Cohen, 2000).

A task-relevant item is one that has motivational value. Motivations, reduced to their primitive dimensions, are either positive/rewarding or negative/punishing. Animal studies or reward have consistently found a relationship between neural activity and DA release along the mesotelencephalic pathway and positively reinforced behavior (Kalivas \& Nakamura, 1999). Human hemodynamic studies have shown similar results, with hemodynamic activity in the VTA, ventral striatum, and medial and orbito-frontal cortex to reward signals (e.g., Knutson, Fong, Bennett, Adams, \& Hommer, 2003; Knutson, Fong, Adams, Varner, \& Hommer, 2001; Breiter \& Rosen, 1999, reviewed in Knutson \& Cooper, 2005). Schultz, Dayan, and Montague (1997) demonstrated that the DA reward system does more than simply respond to reward signals; it codes reward prediction. In their study, when monkeys received an uncued reward, VTA neurons showed the classic reward-sensitive response: enhanced firing at reward delivery. However, if a stimulus that had no intrinsic rewarding properties and did not normally induce VTA firing (e.g., a flash of light) was presented just prior to the reward, after several pairings the VTA neurons began firing to the predictor light (cue) rather than to the reward itself. After conditioning, if the reward predictor was presented but the predicted reward was not delivered, the VTA neurons showed suppressed firing at the time of the expected, but not delivered, reward. Thus, VTA neurons do not simply code reward, they code how reward outcome relates to expectation, showing enhanced firing to an unpredicted reward, when outcome is better than expected, and suppressed firing when a predicted reward is not delivered, when outcome is worse than expected, but no change from baseline when a predicted reward is delivered. This response pattern has been proposed as a mechanism by which motivationally relevant items could gain access to limited-capacity systems (Braver \& Cohen, 2000).

The gate control theory posits that the DA reward prediction system provides an access "gate" to prefrontal neural systems of cognitive control, allowing incoming information to have access to the PFC when the gate is open, but not when the gate is closed (Braver \& Cohen, 2000). The theory posits that one effect of DA on the $\mathrm{PFC}$ is to enhance incoming signal strength by increasing the responsiveness of PFC neurons to afferent projections, thus "opening the gate" when reward expectation is violated (see Cohen et al., 2002; Braver \& Cohen, 2000, for a compete description). If the mesotelencephalic DA system provides a mechanism by which neural representations are selected for preferential processing, then neural indices of attention selection should respond in a manner consistent with DA system neuron responses. Event-related potentials (ERPs) can provide one such neural index of attention selection.
There is a medial frontal positive ERP component at approximately 200-300 msec poststimulus associated with the selection of task-relevant perceptual items, referred to as the frontal selection positivity (FSP) (Kenemans, Kok, \& Smulders, 1993), frontal polar (FP) component (Guillem, Bicu, \& Debruille, 2001), or P2a (Potts, Liotti, Tucker, \& Posner, 1996). This ERP component (referred to here as the P2a) is more positive to stimuli that are instructed targets in the participant's task (see Figure 1). The P2a has the same medial frontal spatial distribution and estimated MFC sources to a variety of target stimuli, including auditory and visual, and in a variety of response tasks, including overt and covert responding, suggesting that it is not sensitive to specific perceptual features or response options but rather to the relevance of the item to the current task (Potts, 2004; Potts, Patel, \& Azzam, 2004; Potts \& Tucker, 2001; Potts, Dien, Hartry-Speiser, McDougal, \& Tucker, 1998). Thus, the spatio-temporal distribution of the $\mathrm{P} 2 \mathrm{a}$ and its cognitive-eliciting conditions are consistent with an index of attention selection in the MFC. The P2a has been associated with the integration of motivational information, specifically reward information from the DA system, with perceptual information for the identification of task-relevant items. However, there is another medial frontal ERP component at the same latency as the P2a, but with opposite voltage polarity, the feedback error-related negativity (ERN) or medial frontal negativity (MFN) that has also been linked to DA input to the MFC, but has been associated with the monitoring of ongoing behavior rather than with attention selection.

The ERN was originally defined as a response-locked component that is more negative on trials on which the

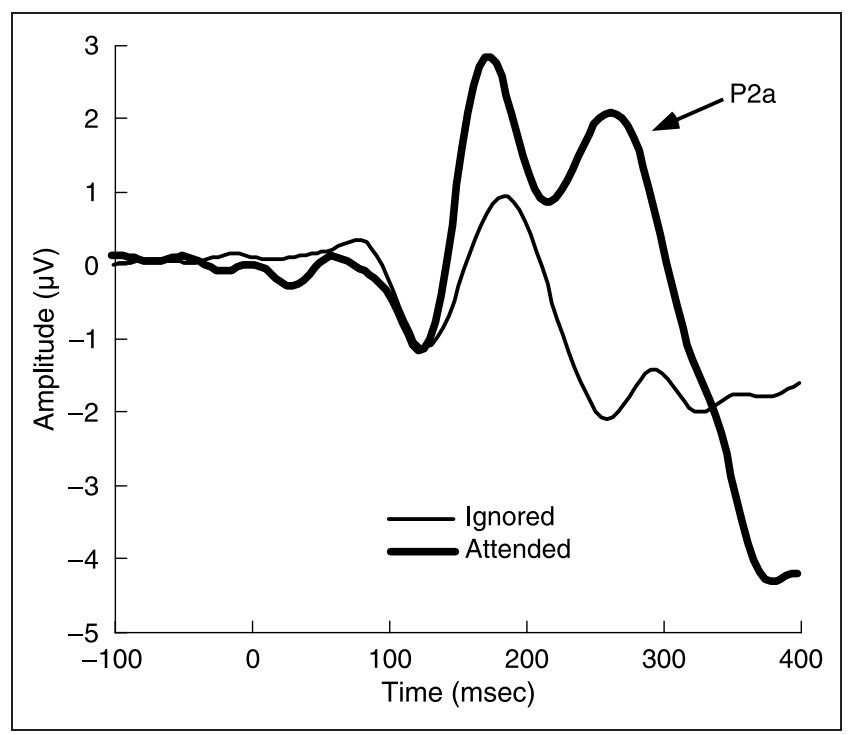

Figure 1. P2a in attention task: prefrontal waveform showing the P2a to attended compared to ignored stimuli (from data published in Potts, Patel, et al., 2004). 
participant made errors (Gehring et al., 1993). Converging evidence from ERP source modeling (Dehaene, Posner, \& Tucker, 1994), hemodynamic imaging (Bush et al., 2002), and single-unit recording (Schall, Stuphorn, \& Brown, 2002) indicates that the MFC is the neural source of the ERN. Although the specific cognitive operation indexed by the ERP is debated, with error detection (Scheffers, Coles, Bernstein, Gehring, \& Donchin, 1996) and mediation between conflicting response options (Gehring \& Fencsik, 2001) being the leading hypotheses, most theories posit that the ERN indexes cognitive processes related to monitoring ongoing behavior (Holroyd \& Coles, 2002).

In some tasks, participants have insufficient information at response execution to evaluate whether their response is correct or not. In those cases, the participants require feedback to know whether their response was an error. In such cases, the ERN is elicited to the feedback rather than the response, with a larger ERN to feedback informing the subject that their response was incorrect (Nieuwenhuis, Holroyd, Mol, \& Coles, 2004). It has not been established whether the response-locked ERN (approximately 0-150 msec after the response) reflects the same neural process as the feedback-locked ERN (about 200-300 msec after the feedback) (Gehring \& Willoughby, 2002b; Holroyd, Coles, \& Nieuwenhuis, 2002), and some authors distinguish between the two by referring to the feedback ERN as the feedback-related negativity (FRN) or MFN (Nieuwenhuis et al., 2004; Gehring \& Willoughby, 2002a). However, both the ERN and MFN localize to the MFC and are larger on error trials, so there is general agreement that they are at least related. In addition, as participants learn which responses are correct and which are incorrect, the error response shifts from the feedback stimulus on early trials to the motor action on later trials, once participants have learned the correct responses, supporting a relationship between the components (Holroyd \& Coles, 2002). Recent findings using monetary incentives have suggested that the MFN may index a more general evaluative function than simple error monitoring.

An explicit error is not required to produce an MFN; an MFN is also elicited in monetarily motivated tasks when the trial outcome is not optimal in the context of the current experiment (Holroyd, Larsen, et al., 2004). Using a task in which participants gained or lost money on a given trial based on their choice between options, Gehring and Willoughby (2002a) found a larger MFN on trials in which the participant's choice on a trial yielded a monetary loss, even though their choice was the best available option on that specific trial (i.e., the "correct" choice). However, the MFN does not appear to index the absolute reward value of an outcome, but rather the value of that outcome relative to the available outcomes, for example, the MFN was largest to breaking even when all other possible outcomes were gains and smallest to the same break-even outcome when all other possible outcomes were losses (Holroyd, Larsen, et al., 2004). Thus, the ERN/MFN appears to index a neural response to actions or the result of actions that fail to meet optimal motivational goals, whether or not that action is incorrect (reviewed in Nieuwenhuis et al., 2004). One model of the ERN/MFN proposes that it indexes a negative reinforcement-learning signal, provided by the DA reward prediction system and applied to a motor representation, when an action or its outcome fails to provide the expected reward value (Holroyd \& Coles, 2002). However, a recent study found an MFN in a passive task, where the participant took no overt actions but the outcome of a computer-generated choice resulted in monetary loss to the participant, indicating that an explicit action is not required to elicit an MFN (Yeung, Holroyd, \& Cohen, 2005). These authors suggest that the MFN may index a more general-purpose motivational evaluation system rather than one tied explicitly to the motor system (Yeung et al., 2005). Because the DA reward prediction system has been theoretically linked to a gate providing access to controlled processing, and actions failing to meet motivational goals might require controlled intervention, this general-purpose system might be related to the same gate that allows access to controlled processing to perceptual representations.

Perceptual items or motor actions that meet motivational expectation can be processed automatically, preserving limited-capacity systems. Items or actions that exceed or fail to meet expectation require additional processing resources to determine why motivational prediction was not met (i.e., the neural representations of those items or actions need preferential processing). Because the MFC receives input from both higher-order perceptual cortex and motor cortex (Van Eden, Lamme, \& Uylings, 1992), and input from the midbrain DA system (Williams \& Goldman-Rakic, 1993), it is anatomically situated to identify representations, either perceptual or motor, that fail to meet motivational expectation. Two ERP components associated with DA reward prediction input to the MFC, the $\mathrm{P} 2 \mathrm{a}$ and the MFN, appear related in that they both occur between about 200 and $300 \mathrm{msec}$, both have medial frontal scalp distributions, and both localize to the MFC. However, the P2a is a positive deflection associated with perceptual item evaluation (Potts, 2004; Potts, Patel, et al., 2004), whereas the MFN is a negative deflection associated with action monitoring (Holroyd et al., 2004; Gehring \& Willoughby, 2002a). If the P2a and MFN index the same medial frontal neural system performing the same generalpurpose evaluative function under the influence of input from the DA reward prediction system, then the response properties of the ERP recorded over medial prefrontal leads between 200 and 300 msec poststimulus should mirror those of VTA DA neurons even in the absence of instructed attention or explicit response: most positive to unpredicted reward and most negative when a predicted reward is not delivered. If the medial 
frontal ERP responds consistently with the VTA neurons, this would support the proposition that the allocation of processing resources to motivationally relevant items, regardless of item type, may be directed by neural systems of reward prediction acting on the MFC.

\section{METHODS}

\section{Participants}

Eighteen undergraduate students at Rice University were paid for their participation in the study. All individuals gave informed consent to participate by written signature.

\section{Experimental Design}

The experiment employed a passive S1/S2 design in which the first stimulus (S1) predicted (and was identical to) the second stimulus (S2) on $80 \%$ of the trials, and S2 delivered the reward value of the trial with $100 \%$ reliability, half rewarding, half not rewarding. The stimuli were images of lemons (S2 reward $=\$ 0$ ) and gold bars (S2 reward $=\$ 1$ ). Thus, on $40 \%$ of the trials, S1 was a lemon and S2 was also a lemon (predicted no-reward condition, outcome $=\$ 0$ ), and on $40 \%$ of the trials, S1 was a gold bar and S2 was also a gold bar (predicted reward, outcome $=\$ 1$ ). However, on $10 \%$ of the trials, S1 was a lemon (predicting no reward), S2 was a gold bar, and the participant received $\$ 1$ (unpredicted reward), and on $10 \%$ of the trials, S1 was a gold bar (predicting a reward), S2 was a lemon, and the participant failed to receive the expected $\$ 1$ (unpredicted noreward condition or predicted reward withheld; see Figure 2 for an example trial). A feedback string informed participants of the outcome of the current trial and their block total. The design was passive; participants were informed of the meaning of the stimuli, but they performed no actions, they simply observed the stimuli. Participants received 480 total trials in eight blocks separated by rest periods, and trial type was randomly selected (constrained by the probabilities described above). Participants began each block with $\$ 5$ in their "bank," and the total dollar amount was reset at the end of each block. At the end of the experiment, participants drew a number between 1 and 8 and were paid in cash their winnings for that block (averaging about $\$ 24)$.

\section{ERP Acquisition and Analysis}

Scalp electroencephalographic data were acquired with a 128-channel EGI system (Electrical Geodesics, Eugene, OR) sampled at $250 \mathrm{~Hz}$, referenced to the vertex with 0.1-100 $\mathrm{Hz}$ analog bandpass filtering, digitally filtered off-line with a $20-\mathrm{Hz}$ low-pass filter, and segmenting offline into 1000-msec epochs spanning $200 \mathrm{msec}$ before to 800 msec after S2. Individual epochs were screened for noncephalic artifact and uncontaminated trials sorted by condition and averaged to create the individual subject ERPs, which were baseline corrected over the 200-msec prestimulus period and transformed into an average reference representation. The individual subject ERPs were averaged together to derive the group central tendency waveform, the grand average. The mean amplitude of the ERP was extracted from frontal leads in the electrode array in a window spanning 200-300 msec poststimulus and cast into a repeated-measures ANOVA with prediction (unpredicted, predicted) and reward (no-reward, reward) as factors.

To isolate the neural responses to outcomes that were better or worse than expected, difference waves were created by subtracting the predicted reward (bar - bar) from the unpredicted no-reward (bar - lemon) waveforms (worse than expected) and the predicted noreward (lemon - lemon) from the unpredicted reward (lemon - bar) waveforms (better than expected) in the grand average data. The ERP scalp topography was plotted and source dipoles were estimated using the BESA program (MEGIS Software, Germany).

\section{RESULTS}

The ERP recorded over medial prefrontal sites was more positive on reward than on punishment trials [Reward: $F(1,17)=14.45, p<.005]$ and was most positive when an unpredicted reward was delivered and most negative

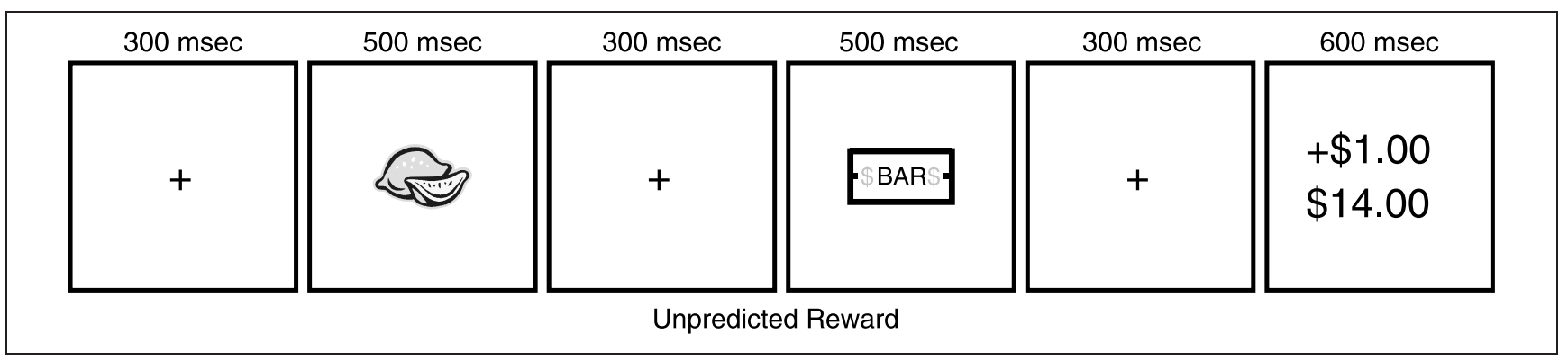

Figure 2. Example trial: trial sequence for an Unpredicted Reward trial, where S1 (lemon) predicts no reward, but S2 (bar) delivers a $\$ 1$ reward on the current trail and a $\$ 14$ running total for the current block, indicated by the feedback string. 
when a predicted reward was not delivered [Reward $x$ Prediction: $F(1,17)=5.70, p<.05$; see Figure 3]. The scalp field topography had a similar medial prefrontal focus for both the better-than-expected (unpredicted reward minus predicted no reward) and worse-thanexpected (unpredicted no reward minus predicted reward) difference waves (see Figure 4). Source modeling of the difference wave produced point dipoles consistent with activity in the MFC, with the better-thanexpected dipole between the right and left anterior cingulate, suggesting bilateral activation, and the worse-than-expected dipole in the right anterior cingulate (Figure 4). A statistical test on vector-length normalized difference-wave amplitudes (normalization is required to test for different neural source configurations between conditions; Picton et al., 2000; McCarthy \& Wood, 1985) across the individual medial frontal region of interest electrodes (excluding the midline electrodes, allowing hemisphere as an explicit factor) revealed no significant effects or interactions with hemisphere or recording site, not allowing a conclusion of different neural source configuration in the two conditions within the centimeter-scale resolution of the ERP.

\section{DISCUSSION}

The ERP between 200 and $300 \mathrm{msec}$ in this passive reward prediction design localized to the MFC, a major target of the mesotelencephalic DA reward system, and the response pattern was consistent with the DA reward system, most positive to unpredicted rewards and most negative when a predicted reward was not delivered. The waveform morphology to unpredicted rewards (Figure 3) was similar to that seen in instructed targets in attention studies (Figure 1) with an initial positive deflection present in both attended and unattended items and in both unpredicted and predicted rewards (larger to the attended items and unpredicted rewards) followed by a second peak, positive to unpredicted rewards (similar to the peak elicited to instructed targets), and a negative deflection when a predicted reward was not delivered. This second peak has the spatio-temporal characteristics of the P2a (positive) and the MFN (negative), with differing polarity depending of outcome valence: positive for better than expected, negative for worse than expected, suggesting that this deflection is related to both the P2a and the MFN.

The P2a was initially described in studies that did not contain explicit rewards, studies that simply had instructions to attend to and respond to one type stimulus, defined by perceptual feature(s), while ignoring other items, and the more positive P2a was interpreted as indexing attention selection of task-relevant perceptual representations (Potts, 2004; Potts \& Tucker, 2001; Potts, Liotti, et al., 1996). The current study had no instruction to attend, but attention might be drawn to unexpected outcomes, those that failed to meet prediction. However, the current design had both better-than-expected and worse-than-expected items, both of which would be selected for attention under the fails-to-meet-prediction
Figure 3. Reward prediction P2a: waveform averaged across the medial prefrontal electrodes (shown in inset) showing the response to an S2 that delivered reward (thick lines) and no-reward (thin lines) when predicted (dashed lines) and unpredicted (solid lines), creating the Predicted Reward (P-R), Predicted No-Reward (P-NR), Unpredicted Reward (U-R), and Unpredicted No-Reward (U-NR) conditions. The P2a window is delineated by vertical dashed lines.

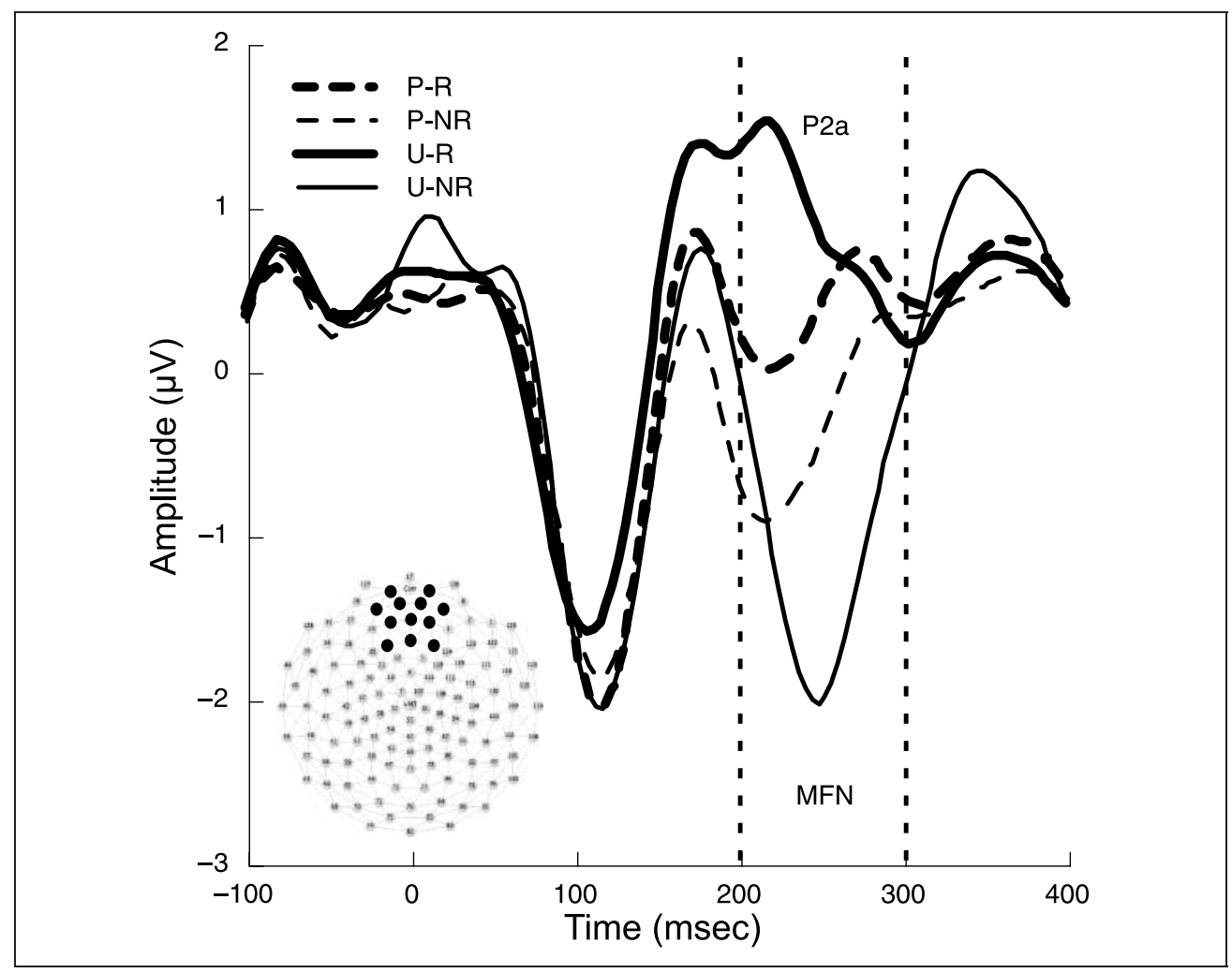




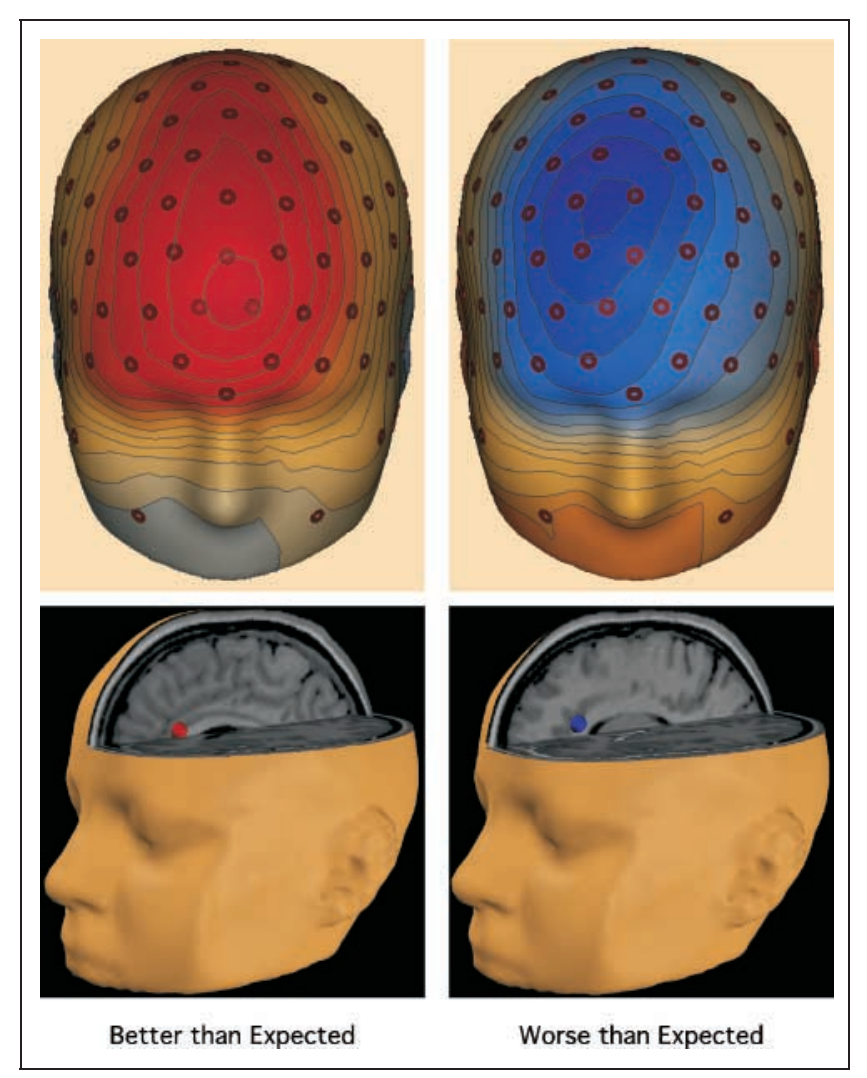

Figure 4. P2a scalp topography and estimated sources: topographic distribution of scalp field distribution and estimated neural sources from the Unpredicted Reward (lemon - bar) minus Predicted No-Reward (lemon - lemon) subtraction (better than expected) and the Unpredicted No-Reward (bar - lemon) minus Predicted Reward (bar - bar) subtraction (worse than expected) difference waves.

criterion, but the better outcome elicited the most positive ERP, whereas the worse outcome elicited the least positive ERP, in fact, a negative peak. Thus, there is an inconsistency between the attention model (attended items elicit a positive $\mathrm{P} 2 \mathrm{a}$ ) and the current findings with a worse-than-expected outcome, requiring attention, producing the least positive deflection, a negativity similar to the MFN.

The MFN has been elicited in designs with explicit rewards (Nieuwenhuis et al., 2004) and has shown similar patterns to the current study, with the worst possible outcomes eliciting the most negative MFN and the best outcomes eliciting the least negative MFN (Holroyd, Larsen, et al., 2004). However, with a single exception (to our knowledge: Yeung et al., 2005), those outcomes have been associated with the execution of overt actions. A leading theory of the MFN links it to the cognitive function of action monitoring, with greater negativity associated with actions that fail to meet motivational goals (Holroyd \& Coles, 2002). The current design, however, was completely passive, containing no response; participants simply observed the appearance of stimuli on the screen and received the predicted and unpredicted rewards (or lack thereof) associated with the stimulus pairings. Thus, there is also an inconsistency between the results from the current passive design and the action monitoring theory of the MFN. Because the P2a and the MFN appear to emanate from the same neural source and can be elicited in the absence of explicit instruction to attend or in the absence of a response, the neural system indexed by these ERP components may perform a more general cognitive process than attention selection or action monitoring, a process that contributes to both of those operations. Providing access of information to neural systems of cognitive control by computing the motivational value of currently available neural representations, whether of perceptual items or of motor actions, is one operation that contributes to both attention selection and action monitoring.

Most perceptual representations can be processed automatically, without controlled intervention. Likewise, executing most actions does not require higher-level conscious control. As long as environmental items and the outcomes of actions meet motivational expectation, no higher-level controlled intervention is requiredprocessing can proceed automatically, sparing limitedcapacity systems. However, if some item or action produces an outcome that either exceeds or fails to meet the current predicted motivational result, then those item or action representations need access to higher-order systems of cognitive control for additional processing. The anatomical connections of the medial prefrontal cortex would allow this function.

The MFC receives input from higher-order perceptual and motor cortex as well as from the midbrain DA system (Williams \& Goldman-Rakic, 1993; Van Eden et al., 1992), and therefore has access to both elaborated perceptual and motor representations, as well as to reward prediction information. Thus, the MFC can integrate perceptual and motor representations with information about how current outcome meets expected outcome to identify both perceptual objects and action plans that do not meet motivational expectation. The DA system can produce both positive and negative reward prediction-related responses, signaling a motivational state that is either better or worse than expected, and provide this signal to the MFC, which has assess to both perceptual and motor representations. Thus, the MFC can identify the motor or perceptual representations that are present when an outcome prediction is violated. The valence of this prediction error is coded by change in firing rate of DA source neurons in the VTA, with better-than-predicted outcomes coded by increased firing rate and worse-than-predicted outcomes coded by decreased firing rate. This valence coding appears to be maintained in the medial prefrontal target of these DA projections, as indexed by the polarity of the $\mathrm{P} 2 \mathrm{a} / \mathrm{MFN}$.

The general model relating EEG and ERP polarity to neural activity holds that the scalp-recorded waveforms 
arise from inhibitory and excitatory postsynaptic potentials (IPSPs and EPSPs, respectively) in the apical dendrites of cortical pyramidal cells summed across a contiguous cortical sheet (Nunez \& Srinivasan, 2005). Scalp positive deflections are thought to be due to either IPSPs at deep cortical layers or EPSPs at shallow layers, whereas scalp negative deflections are due to deep EPSPs or shallow IPSPs, with shallow input coming from cortical-cortical projections and deep input coming from subcortical-cortical projections. Holroyd and Coles (2002) proposed that the ERN negativity results from deep-layer EPSPs resulting from disinhibition caused by reduced DA input when outcomes are worse than predicted. By the same logic, a surface positivity would result from deep-layer inhibition from increased DA input when the outcome is better than predicted.

The current results do not address how enhanced and suppressed DA input to the MFC translates into access to neural systems of cognitive control in the prefrontal cortex. Gate control theory posits a direct effect of DA on the PFC, increasing sensitivity of PFC neurons to incoming signals, whereas the current results indicate mediation by the MFC. The neurophysiology of this mediation remains to be described but may include medial prefrontal connections to the dorsolateral prefrontal cortex, the orbito-frontal cortex, and the ventral striatum. The current results are consistent with midbrain DA mediated selection in the MFC. The medial frontal ERP to better- and worse-than-predicted outcomes had a similar spatio-temporal distribution, indicating that this ERP reflects activity in the MFC, with positive polarity to better-than-expected outcomes and negative polarity to worse-than-expected outcomes. This response pattern is consistent with neuron firing patterns in the midbrain DA reward prediction system, which show enhanced firing to unpredicted rewards and suppressed firing when a predicted reward is not delivered. Thus, the P2a, which was previously associated with the task-relevance evaluation of perceptual items, and the MFN, which was previously associated with the monitoring of ongoing behavior, may reflect the same index of activity in a neural system for the integration of action and/or perceptual representations with motivational state information, identifying items and actions associated with reward prediction violations. The MFC may provide a gate through which both perceptual and motor representations gain access to preferential processing. Through this MFC evaluation system, prefrontal limited-capacity systems of cognitive control can be allocated to the most salient items and actions currently available, items that exceed or fail to meet motivational expectation.

\section{Acknowledgments}

This study was supported by R01 DA14073 (Potts), R01 DA11723 (Montague), and R01 NS045790 (Montague).
Reprint requests should be sent to Geoffrey F. Potts, Rice University, Psychology MS-25, P.O. Box 1892, Houston, TX 770051892, or via e-mail: gpotts@rice.edu.

\section{REFERENCES}

Braver, T. S., \& Cohen, J. D. (2000). On the control of control: The role of dopamine in regulating prefrontal function and working memory. In S. Monsell \& J. Driver (Eds.), Attention and performance XVIII (pp. 713-738). Cambridge: MIT Press.

Breiter, H. C., \& Rosen, B. R. (1999). Functional magnetic resonance imaging of brain reward circuitry in the human. In J. F. McGinty (Ed.), Advancing from the ventral striatum to the extended amygdala: Implications for neuropsychiatry and drug use: In honor of Lennart Heimer Annals of the New York Academy of Sciences, Vol 877 (pp. 523-547). New York: New York Academy of Sciences.

Bush, G., Vogt, B. A., Holmes, J., Dale, A. M., Greve, D., Jenike, M. A., \& Rosen, B. R. (2002). Dorsal anterior cingulate cortex: A role in reward-based decision making. Proceedings of the National Academy of Sciences, U.S.A., 99, 523-528.

Cohen, J. D., Braver, T. S., \& Brown, J. W. (2002). Computational perspectives on dopamine function in prefrontal cortex. Current Opinion in Neurobiology, 12, 223-229.

Dehaene, S., Posner, M. I., \& Tucker, D. M. (1994). Localization of a neural system for error detection and compensation. Psychological Science, 5, 303-305.

Gehring, W. J., \& Fencsik, D. E. (2001). Functions of the medial frontal cortex in the processing of conflict and errors. Journal of Neuroscience, 21, 9430-9437.

Gehring, W. J., Goss, B., Coles, M. G., Meyer, D. E., \& Dochin, M. (1993). A neural system for error detection and compensation. Psychological Science, 4, 385-390.

Gehring, W. J., \& Willoughby, A. R. (2002a). The medial frontal cortex and the rapid processing of monetary gains and losses. Science, 295, 2279-2282.

Gehring, W. J., \& Willoughby, A. R. (2002b). "Medial prefrontal cortex and error potentials": Response. Science, 296, 1611.

Guillem, F., Bicu, M., \& Debruille, J. B. (2001). Dissociating memory processes involved in direct and indirect tests with ERPs to unfamiliar faces. Cognitive Brain Research, 11, 113-125.

Holroyd, C. B., \& Coles, M. G. (2002). The neural basis of human error processing: Reinforcement learning, dopamine, and the error-related negativity. Psychological Review, 109, 679-709.

Holroyd, C. B., Coles, M. G., \& Nieuwenhuis, S. (2002). Medial prefrontal cortex and error potentials. Science, 296, 1610-1611.

Holroyd, C. B., Larsen, J. T., \& Cohen, J. D. (2004). Context dependence of the event-related brain potential associated with reward and punishment. Psychophysiology, 41, 245-253.

Kalivas, P. W., \& Nakamura, M. (1999). Neural systems for behavioral activation and reward. Current Opinion in Neurobiology, 9, 223-227.

Kenemans, J. L., Kok, A., \& Smulders, F. T. (1993). Eventrelated potentials to conjunctions of spatial frequency and orientation as a function of stimulus parameters and response requirements. Electroencephalography and Clinical Neurophysiology, 88, 51-63. 
Knutson, B., \& Cooper, J. C. (2005). Functional magnetic resonance imaging of reward prediction. Current Opinion in Neurology, 18, 411-417.

Knutson, B., Fong, G. W., Adams, C. M., Varner, J. L., \& Hommer, D. (2001). Dissociation of reward anticipation and outcome with event-related fMRI. NeuroReport, 12, 3683-3687.

Knutson, B., Fong, G. W., Bennett, S. M., Adams, C. M., \& Hommer, D. (2003). A region of mesial prefrontal cortex tracks monetarily rewarding outcomes: Characterization with rapid event-related fMRI. Neuroimage, 18, 263-272.

McCarthy, G., \& Wood, C. C. (1985). Scalp distributions of event-related potentials: An ambiguity associated with analysis of variance models. Electroencephalography and Clinical Neurophysiology, 62, 203-208.

Miller, E. K., \& Cohen, J. D. (2001). An integrative theory of prefrontal cortex function. Annual Review of Neuroscience, 24, 167-202.

Nieuwenhuis, S., Holroyd, C. B., Mol, N., \& Coles, M. G. H. (2004). Reinforcement-related brain potentials from medial frontal cortex: Origins and functional significance. Neuroscience and Biobehavioral Reviews, 28, 441-448.

Norman, D. A., \& Shallice, T. (1986). Attention to action: Willed and automatic control of behavior. In R. J. Davidson, G. E. Schwartz, \& D. Shapiro (Eds.), Consiousness and self-regulation (pp. 1-18). New York: Plenum Press.

Nunez, P. L., \& Srinivasan, R. (2005). Electric fields of the brain: The neurophysics of EEG (2nd ed.). New York: Oxford University Press.

Parasuraman, R. (Ed.) (1998). The attentive brain. Cambridge: MIT press.

Picton, T., Bentin, S., Berg, P., Donchin, E., Hillyard, S., Johnson, R., Jr., Miller, G. A., Ritter, W., Ruchkin, D. S., Rugg, M. D., \& Taylor, M. J. (2000). Guidelines for using human event-related potentials to study cognition: Recording standards and publication criteria. Psychophysiology, 37, 127-152.

Posner, M. I., \& Dehaene, S. (1994). Attentional networks. Trends in Neurosciences, 170, 75-79.

Posner, M. I., \& Petersen, S. E. (1990). The attention systems of the human brain. Annual Review of Neuroscience, 13, 25-42.
Potts, G. F. (2004). An ERP index of task relevance evaluation of visual stimuli. Brain \& Cognition, 56, 5-13.

Potts, G. F., Dien, J., Hartry-Speiser, A. L., McDougal, L. M., \& Tucker, D. M. (1998). Dense sensor array topography of the event-related potential to task-relevant auditory stimuli. Electroencephalography and Clinical Neurophysiology, 106, 444-456.

Potts, G. F., Liotti, M., Tucker, D. M., \& Posner, M. I. (1996). Frontal and inferior temporal cortical activity in visual target detection: Evidence from high spatially sampled event-related potentials. Brain Topography, 9, 3-14.

Potts, G. F., Patel, S. H., \& Azzam, P. N. (2004). Impact of instructed relevance on the visual ERP. International Journal of Psychophysiology, 52, 197-209.

Potts, G. F., \& Tucker, D. M. (2001). Frontal evaluation and posterior representation in target detection. Cognitive Brain Research, 11, 147-156.

Schall, J. D., Stuphorn, V., \& Brown, J. W. (2002). Monitoring and control of action by the frontal lobes. Neuron, 36, 309-322.

Scheffers, M. K., Coles, M. G. H., Bernstein, P., Gehring, W. J., \& Donchin, E. (1996). Event-related brain potentials and error-related processing: An analysis of incorrect responses to go and no-go stimuli. Psychophysiology, 33, $42-53$.

Schultz, W., Dayan, P., \& Montague, P. R. (1997). A neural substrate of prediction and reward. Science, 275, 1593-1599.

Van Eden, C., Lamme, V., \& Uylings, H. (1992). Heterotopic cortical afferents to the medial prefrontal cortex in the rat. A combined retrograde and anterograde tracer study. European Journal of Neuroscience, 4, 77-97.

Williams, S. W., \& Goldman-Rakic, P. S. (1993). Characterization of the dopaminergic innerfaction of the primate frontal cortex using a dopamine-specific antibody. Cerebral Cortex, 3, 199-222.

Yeung, N., Holroyd, C. B., \& Cohen, J. D. (2005). ERP correlates of feedback and reward processing in the presence and absence of response choice. Cerebral Cortex, 15, 535-544. 